\title{
Hierarchical Compressed Sensing for Cluster Based Wireless Sensor Networks
}

\author{
Vishal Krishna Singh \\ Dept. of Information Technology \\ Indian Institute of Information Technology \\ Allahabad, India
}

\author{
Manish Kumar \\ Dept. of Information Technology \\ Indian Institute of Information Technology \\ Allahabad, India
}

\begin{abstract}
Data transmission consumes significant amount of energy in large scale wireless sensor networks (WSNs). In such an environment, reducing the in-network communication and distributing the load evenly over the network can reduce the overall energy consumption and maximize the network lifetime significantly. In this work, the aforementioned problem of network lifetime and uneven energy consumption in large scale wireless sensor networks is addressed. This work proposes a hierarchical compressed sensing (HCS) scheme to reduce the innetwork communication during the data gathering process. Corelated sensor readings are collected via a hierarchical clustering scheme. A compressed sensing (CS) based data processing scheme is devised to transmit the data from the source to the sink. The proposed HCS is able to identify the optimal position for the application of CS to achieve reduced and similar number of transmissions on all the nodes in the network. An activity map is generated to validate the reduced and uniformly distributed communication load of the WSN. Based on the number of transmissions per data gathering round, the bit-hop metric model is used to analyse the overall energy consumption. Simulation results validate the efficiency of the proposed method over the existing CS based approaches.
\end{abstract}

Keywords-Compressed sensing; in-network communication; network lifetime; traffic load balancing; wireless sensor network

\section{INTRODUCTION}

Wireless sensor networks (WSNs) have revolutionised today's practice of numerous scientific and engineering endeavours, including ecosystems, environmental sciences, military applications, scientific research etc. WSNs are used for sensing physical variables of interest at unprecedented high spatial densities and long-time durations [1]. Applications like environmental monitoring, scientific research etc., explore the benefits of WSNs. Such applications require transferring a huge amount of sensed data from one point of the network to another. Considering the fact, that the energy consumed in transmission of $1 \mathrm{~Kb}$ of data over a distance of 100 meters is equal to the energy consumed in executing 300 million instructions with the rate of computation being 100 million instructions per second on a processor with general configurations [3], [4]. Almost $70 \%$ of the total energy is consumed in communication within the network [2]. Hence, the inherent constraints of WSNs such as limited bandwidth and limited battery life makes them prone to failure and compromise the network lifetime. Significant energy conservation in such networks can be achieved by: a) minimizing the cost of interaction between the nodes and $b$ ) achieving traffic load balancing during in-network communications [3]. Techniques such as data aggregation have been used to efficiently reduce the communication load of the network, however the issue of asymmetric load distribution in the network remain an important concern till date. Load balancing and optimized energy consumption are thus, much sought after parameters for multi hop data transmission in WSNs.

This work addresses the problem of uneven energy consumption and network lifetime maximization through a novel in-network data processing scheme which incorporates $\mathrm{CS}$ in a novel way over a clustered routing structure. The nodes are randomly deployed in a sensing area which is divided into homogenous sub-regions. Such a division is done to model the real world scenario of an area such as a thermal power plant. In such a deployment, the area can be divided into homogeneous regions in such a way that one homogeneous region is different from the other.

For example, the area with the thermal station (one homogeneous region) will exhibit high temperature readings as compared to the residential areas of the plant (another homogeneous region) and are known as a priori. The proposed HCS is divided into two phases, a) Clustering and communication phase b) CS and data processing phase. The proposed scheme incorporates CS in a way to efficiently distribute the communication load evenly over the network. To the best of our knowledge, the advantages of using CS and hybrid CS on tree based routing structure are many but its advantages over a clustered routing structure have not been explored yet. The contributions of this work can be summarised as:

- A CS based data processing scheme with minimum innetwork transmissions.

- A scheme for enhancing the network lifetime by balanced network traffic load distribution

The remainder of the paper is organised as follows: in section II, a summary of the related work is discussed. In section III the problem statement for the proposed work is given. The section IV presents the proposed scheme along with the detailed analysis and framework for CS. A detailed analysis of the energy consumption, based on the bit hop metric model, is also discussed. Finally, in section V, a detailed explanation of the simulation results is presented. The section VI summarises the proposed work with concluding comments. 


\section{RELATED WORK}

Data aggregation in WSNs is considered to be the most easily deployable data reduction technique [2]. With varying network topology, such as cluster based [2], tree based [3], chain based [4], various data aggregation schemes have been proposed [4], [5], [6], [7], [8], [9]. Data aggregation mainly exploits the redundancy in the spatially and temporally corelated data sensed by the nodes [10]. Hence, significant energy conservation is achieved by reducing the amount of data being forwarded by any node. However, data aggregation approaches suffer from certain disadvantages. The most important concern with most of the data aggregation approaches, is the loss of information. Data aggregation approaches mainly focus on transferring only a summary of the sensed value to the sink. Hence, a lot of information about the measured value is sacrificed by the aggregation techniques [11]. Another drawback of data aggregation schemes is the asymmetric load distribution within the network. This results in parts of the network having relatively higher activity and thus becoming non-operational because of dead nodes, leaving the sink isolated. For example, in case of an event, WSNs have large amount of data flowing in the network. In such an environment, activity in the deployment area depends upon the occurrence of an event and position of the nodes [12]. The nodes near the sink have high energy consumption as compared to the nodes in other region because of heavy load of data transmission to the sink. Asymmetric distribution of the load results in high activity in parts of the network causing nodes with heavy communication load to die quickly.

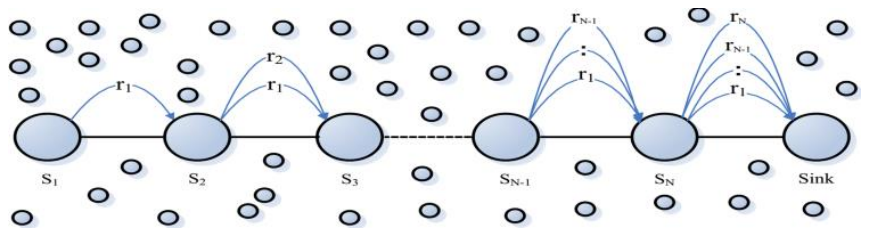

Fig. 1. Traditional data gathering in multi-hop environment

CS has evolved as a promising technique, which can efficiently overcome the drawbacks of the existing aggregation approaches. Data gathering in traditional multi hop environment can be understood from the Fig 1. which shows the highlighted path in a WSN where, 'N' sensor nodes form a multi-hop path for data collection. Let the reading generated by the node S1 is r1. Similarly, reading generated by the node S2 is $\mathrm{r} 2$ and so on. In a normal data acquisition process, the node $\mathrm{S} 1$ sends its reading to node $\mathrm{S} 2$. S2 in turn transmits both its data $\mathrm{r} 2$ and the data obtained from S1 to S3 .Finally in the end, the last node of the route, sends all the data received from previous nodes along with its own data to the sink. As seen in the Fig.1, the nodes closer to the sink consume more energy as compared to the nodes away from the sink. Due to this, the nodes closer to sink will be drained quickly compromising the lifetime of the network.

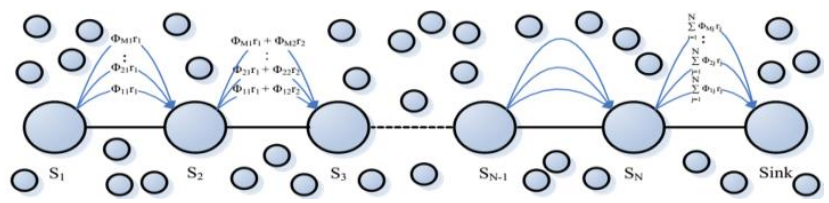

Fig. 2. Data gathering with Compressed Sensing
The Fig. 2 shows the compressed data gathering method in the highlighted path of a WSN where, ' $N$ ' sensor nodes form a multi-hop path for data collection. The sink upon receiving all the $\mathrm{M}$ samples from ' $\mathrm{N}$ ' nodes, reconstructs the original data. In order to send the $\mathrm{i}^{\text {th }}$ sample to the sink, $\mathrm{S} 1$ generates a random coefficient $\Phi_{i 1}$ and multiplies it's reading i.e. r1 with it. The product is then sent to the node S2. Similar multiplication is performed at the node $\mathrm{S} 2$ with the random coefficient $\Phi_{i 2}$ and the $\operatorname{sum} \Phi_{i 1} r_{1}+\Phi_{i 2} r_{2}$ is sent to the node S3. This process is followed by every node in the route to send M samples of their data. Such a transmission results in the sink receiving $\sum_{j=1}^{n} \Phi_{i j} r_{j}$. The number of samples sent by each node is limited to M. Comparing the Fig.1 and Fig.2, three observations can be made: (a) all the nodes in compressed data gathering, perform the same number of computations (b) the number of transmissions of the first $M$ nodes is more in compressed data gathering scheme as compared to normal transmissions, but the remaining nodes send less messages(c) compressed data gathering scheme distributes the load equally among the nodes of the routing path. Since $M$ is a much smaller number as compared to $\mathrm{n}$, this becomes clearly visible that the number of transmissions in compressed data gathering scheme is far less than normal data gathering [12]. Although, the number of transmissions for collecting $\mathrm{M}$ samples from $\mathrm{N}$ node is reduced to $\mathrm{MN}$, the literature supports the fact that applying CS naively might not be as beneficial as applying it on a later stage. Considering the drawbacks of applying CS at initial stages of the data gathering process, hybrid approaches were proposed. The hybrid CS scheme proposed in [13] allows the leaf node to sense and send the data without using the CS method, but the nodes which are closer to the sink are the ones which are responsible for the application of CS. The approach in [13] proposes a threshold value K, beyond which CS scheme should be applied but before the threshold is reached, the data collection proceeds in a normal fashion. Owing to the fault tolerance and optimal load balancing properties of clustered routing, the tree based routing scheme becomes the secondary choice of routing strategy in this work. One other advantage of clustered routing is better traffic balancing, which makes the clustering approach preferable choice over the tree based routing scheme. A theoretical analysis presented in [14] proves the efficiency of the compressed data gathering. Aiming the network energy consumption, the authors in [15] proposed a greedy heuristic solution which uses CS with joint routing protocol. In [16], Zigbee protocols are considered in a wireless sensor environment with the assurance of reduced energy consumption. The authors proposed an adaptive scheme which uses CS to reduce the number of transmissions over the network. The scheme proposed in [17], makes use of data aggregation trees formed by subdividing the sensor network into sub networks. The authors in [18], [19] described a three phased CS data gathering scheme. The sensing region is classified into cells with a unique cell head. The cell head collects and forwards the data in compressed fashion along the columns to the Vth row. Finally the data is relayed to the sink along the Vth row cells. In a recent work in [20], the authors proposed a clustering based compressed data gathering 
scheme. The authors divide the nodes in a clustered fashion to transmit the compressed data through various levels.

Thus, variations of CS have proved to be advantageous in such an environment as WSN, but the existing approaches have certain disadvantages. An important concern is that most of the existing work on CS view compression from the signal processing perspective only [21], [22]. Applications on data compression, from the networking protocol perspective in WSNs are limited [23]. CS, if and when applied naively to a sensor network, imposes extra burden over the network. As shown in Fig. 2, suppose $N-1$ nodes are each sending one sample to the Nth node, the outgoing link of that node will carry ' $\mathrm{N}$ ' samples if no aggregation is performed; or will carry 1 sample if lossy aggregation is performed. If we apply the CS principle directly, the CS aggregation will force every link to carry ' $M$ ' samples, leading to unnecessary higher traffic at the early stage transmissions [13]. To overcome these drawbacks, the idea of hybrid CS was proposed but hybrid CS has its own disadvantages. The selection of non-CS and CS points within the hybrid-CS scheme is critical in getting the benefit of CS [13], [14], [15]. Distributed CS [23] suffers as compared to a mixed protocol in large-scale WSNs, under real technological constrains. Unless the network size and compression are both taken into consideration in network design, distributed CS approaches tend to have average performance in terms of lifetime and energy conservation. Interestingly, existing works on compressed data gathering in WSNs, mainly exploit the tree based routing structure. Because of the drawbacks of the tree based routing structure, such as unstable network topology, most of the existing works face the problem of unreliability and poor quality of service [26].

Thus, the proposed HCS is developed on the principle of hybrid CS over clustered routing structure, with the aim of achieving reliable data transmission with minimum energy consumption (achieved by minimizing and balancing the network traffic evenly over the network).

\section{PROBLEM DEFINITION}

The main objective in WSNs is to reduce the in-network communication and improve the throughput of the network by increasing the network lifetime. However, network lifetime in large scale wireless sensor networks is also significantly affected by uneven energy expenditure by sensor nodes. Nodes with heavy communication load consume more energy and die quickly causing holes and isolation of some regions of the network. It is therefore desirable to process as much data locally as possible so as to reduce the number of bits transmitted. Techniques such as data aggregation are used to reduce the amount of data being forwarded by the nodes. However, data aggregation schemes have certain drawbacks such as, asymmetric load distribution and information loss. In case of an event, WSNs have large number of message transmissions in the network. In such an environment, activity in the deployment area depends upon the occurrence of an event and position of the nodes. The nodes near the sink have high energy consumption as compared to the nodes in other regions because of heavy load of data transmission to the sink. An asymmetric distribution of the load results in parts of the network having relatively higher activity and thus becoming non-operational because of dead nodes, leaving the sink isolated. Energy conservation may be achieved by transmitting only the summary of the sensed data that may result loss of information. Recent reported work state that, the idea of using CS for data transmission can be advantageous in the above scenario. Naïve application of CS to a sensor network imposes extra burden over the network, hence Hybrid CS would be the most suited solution. However, determining the CS and nonCS points is crucial in such approaches to explore the true potential of the scheme. Hence a CS scheme with the ability of uniform load distribution and efficient data transmission is desired. Reduced in-network communication and optimized energy usage in such a scheme will significantly improve stability period and reduced instable region. Hence, a scheme based on compressed sensing over clustered routing structure is proposed.

\section{PROPOSED APPROACH}

\section{A. Compressed sensing}

The idea of compressive data gathering is relatively new in the field of wireless sensor networks. Some of the basic yet essential properties of the framework asserts that, a relatively small number of samples of a sparse data contains enough information to successfully recover the original data with almost no data loss [27]. Mathematically, If a sparse data ' $x$, can be denoted as $x=\left\{x_{1}, x_{2}, x_{3} \ldots \ldots x_{N}\right\}^{\mathrm{T}}$ such that $x \in \mathrm{R}^{N}$ and the orthogonal sparse basis or projection of $x$ is given by $\Psi=\left[\Psi_{1}, \Psi_{2}, \Psi_{3} \ldots \ldots . \Psi_{N}\right]$ where $\Psi_{i}$ is the $i^{\text {th }}$ column of $\Psi$, then $x$ can be given by the following equation:

$$
x=\Psi S=\sum_{i=1}^{N} \Psi_{i} \cdot S_{i}
$$

Such that, $\mathrm{S}$ is a vector of the coefficient matrix $\Psi$ and "." represents the inner product. According to the theory of the CS, provided the target data $x$ is $\mathrm{K}$-sparse in the basis $\Psi$, then under specific conditions, $M$ adaptive measurements of $x$ are sufficient to fully recover the original data such that $M<<\mathrm{N}$. Where each weighted measurement, $\mathrm{y}$, can be written as:

$$
y=\Phi x
$$

The recovery of the target data by $\mathrm{M}$ measurements is dependent on the following condition:

$$
M \geq c . \mu^{2}(\Phi \Psi) . K \cdot \log N
$$

Where $\Phi$ is a $M \times N$ sensing matrix, 'c' is a positive constant and $\mu(\Phi \Psi)$ is the coherence between the sensing matrix $\Phi$ and the representation basis $\Psi$. The original data is recovered by solving a convex optimization problem, given by:

$$
\begin{aligned}
& \left(\|s\| l 1=\sum_{i}\left|s_{i}\right|\right) \\
& \begin{array}{c}
\min \\
\text { sò }^{n}\|s\| l 1
\end{array} \\
& \text { provided, } y=\Phi \Psi S
\end{aligned}
$$


And considering, $x=\Psi \hat{s}$, with $\hat{s}$ being the optimal solution.

Thus, an important conclusion can be drawn from the assertion is that the basic foundation of CS relies on sensing matrices. For reliable and efficient compression, the data must be sparse in some intitutively known domain and the sensing matrix, $\Phi$, must meet the restricted isometric property (RIP).

An essential property of the sensing matrix, $\Phi$, is the Null space property (NSP) and is denoted by:

$$
N(\Phi)=\{z: A z=0\} \text {. NSP }
$$

To explain, a sparse data $\mathrm{x}$, can be completely recovered by $\Phi \mathrm{x}$, if for every pair of distinct vectors such as $x, x^{\prime} \in \sum_{k}$, $\Phi \mathrm{x}=\Phi \mathrm{x}^{\prime}$. Considering the mentioned condition is not true i.e. if $\Phi \mathrm{x}=\Phi \mathrm{x}^{\prime}$, results $\Phi\left(x-x^{\prime}\right)=0$ such that $x-x \in \sum_{2 k}$. Therefore, it can be said that for 'A' to uniquely represent every $x \in \sum_{k}, N(\Phi)$ should not contain a vector that belongs to $\sum_{2 k}$. This feature of the sensing matrix is known as spark of the matrix and is defined as:

Definition 1. The spark of a sensing matrix given by $\Phi$, is the smallest number of linearly dependent columns of $\Phi$.

Theorem 1. If the spark of a sensing matrix $\Phi$, is greater than $2 k$ then, for a given vector, $\mathrm{y}$, where $y \in \mathrm{R}^{m}$, there exists a unique data $x \in \sum_{k}$, such that $y=\Phi x$.

Proof: To prove the theorem 1 by contradiction, let there be a vector ' $\mathrm{y}$ ' such that $y \in \mathrm{R}^{m}$ and there exists a unique data $x \in \sum_{k}$, such that $y=\Phi x$. It is assumed that, spark $(\Phi) \leq 2 k$. Or it can be said that there are at most $2 k$ linearly independent columns which implies that there is an $h \in N(\Phi)$ such that $h \in \sum_{2 k}$. Now, since $h \in \sum_{2 k}$, therefore $h=x-x^{\prime}$, where $x, x^{\prime} \in \sum_{k}$. As we know that $h \in N(\Phi)$, therefore $\Phi\left(x-x^{\prime}\right)=0$ and $\Phi x=\Phi x^{\prime}$. But this is a contradiction of the above assumption that there exists a unique data $x \in \sum_{k}$, such that $y=\Phi x$. Hence, spark $(\Phi)>2 k$. Now, considering spark $(\Phi)>2 k$, and for a given ' $y$ ' there exist $x, x^{\prime} \in \sum_{k}$, such that: $\Phi=\Phi x=\Phi x$ '. This implies that, $\Phi\left(x-x^{\prime}\right)=0$, OR $\Phi \mathrm{h}=0$, replacing $x-x^{\prime}$ with $h$.
Since $\operatorname{spark}(\Phi)>2 k$, hence at most $2 k$ columns of $\Phi$ are linearly independent and $h=0$. Therefore, the theorem 1 is proved as $x=x^{\prime}$. An important conclusion from the above theorem is that the number of measurements i.e. $m$, should follow the following condition:

\section{$m \geq 2 k$}

Definition 2. For a measurement matrix $\Phi$, to satisfy the null space property (NSP) of order $k$, there must exist a constant $C>0$ such that,

$$
\left\|h_{\wedge}\right\|_{2} \leq C \frac{\left\|h_{\wedge}\right\| 1}{\sqrt{k}}
$$

is true for all $h \in N(\Phi)$ and for all ' ' such that $|\wedge| \leq k$.

That is, a k-sparse vector in $N(\Phi)$ is $h=0$, iff, the matrix $\Phi$ satisfies the NSP. The literature supports the fact that a NSP of order $2 k$ is necessary and sufficient condition for a recovery algorithm (say $l 1$ minimization).

The NSP guarantees do not cover for data, which is degraded because of noise. In [27], the authors proposed RIP on the sensing matrix ' $\Phi$ ' for full recovery of the data even if it is corrupted.

Thus, the framework for data transfer using hierarchical CS in WSN can be summarised with the following advantages:

1) The computation load is shifted from encoder $(\mathrm{CH})$ to the decoder end (sink).

2) Routing for data transmission is independent of the compression.

3) Same number of data packets for every node in the network.

Considering the above advantages, an efficient data transfer scheme for wireless sensor networks is proposed in $C$ and $D$.

\section{B. Sensor Network Model}

The graph, $G=\langle\mathrm{V}, E\rangle$, is the sensor network where $\mathrm{V}$ consists of all $(\mathrm{N})$ sensor nodes in the network and the sink node given by $v_{0}$. A link is assumed to be present between two nodes of $\mathrm{V}$ iff, the two nodes are within each other's communication range. A hierarchical clustering scheme, with at most two hop transmission, is used for the cluster formation. The nodes choose a random number between 0 and 1 and compare it to the threshold broadcasted by the sink. The nodes with values higher than the threshold are chosen as the cluster head. A major concern of hybrid CS is the point of application of CS and non CS strategies. In this work, the true potential of hybrid CS is explored by choosing two optimal points for the application of CS. Specifically, CS is applied at the FCHs and at the one hop neighbours of the FCHs, depending upon the transmissions received at both the points. Finally, Huffman encoded data is obtained at the $\mathrm{SCH}$ and is transmitted to the sink. 
A network model with the following assumptions is considered:

1) A wireless sensor network is randomly deployed and the sensor nodes transmit the data on the occurrence of an event.

2) The network consists of only one sink.

3) The deployed area is randomly divided into subregions in such a way that readings from one region are different from the other.

4) Establishing routing information consumes relatively less energy as compared to the data gathering process, hence it is not considered for energy computation.

The two phases of the proposed scheme are discussed in the following sections:

\section{Clustering and Communication}

Selection of First level cluster head (FCH)

The FCH is chosen using the standard LEACH protocol from the deployed sensor nodes. Once the FCHs have been identified, the cluster formation and communication protocol is established as follows:

1) The FCH sends a join message to all the one hop neighbours.

2) All the one hop neighbours join the FCH if one of the following condition is true:-

$\checkmark$ It has not received a join message from any other FCH.

$\checkmark$ It has received a join message from more than one $\mathrm{FCH}$. In this case the node joins the nearest FCH.

3) One hop neighbours, after joining the $\mathrm{FCH}$, follow the two hop communication protocol and broadcast a join message to their one hop neighbours.

4) The two hop neighbours of the FCH follow the same communication protocol as explained in (ii).

5) The data is compressed using CS and is forwarded to respective SCHs.

Selection of Second level cluster head (SCH)

Assuming that the sink is aware of the position of all the nodes including the FCHs, the FCHs closest to the sink are identified as the SCHs. A multicast message from the sink to all the FCHs establishes the communication hierarchy. The communication protocol is established as follows:

6) The SCH sends a join message to all the one hop FCHs.

7) All the one hop FCHs join the SCH if one of the following condition is true:-

$\checkmark$ It has not received a join message from any other $\mathrm{SCH}$.

$\checkmark$ It has received a join message from more than one $\mathrm{SCH}$. In this case the node joins the nearest $\mathrm{SCH}$.

8) One hop FCHs, after joining the SCH, follow the two hop communication protocol and broadcast a join message to their one hop neighbours.

9) Two hop neighbours of the SCH follow the same communication protocol as explained in (step 7).
10) The SCH applies Huffman encoding on the received data and forwards it to the sink.

\section{Compressed Sensing and Data processing}

The compression ratio $(u)$ is defined as the ratio between the amount of data available for transmission and the the amount of data actually transmitted. In a large scale WSN, the number of nodes in a cluster can be relatively high, leading to large amount of data at the cluster heads (CHs). Applying CS at the $\mathrm{CH}$ reduces the number of bits significantly, but the compression ratio might still be very low due to huge amount of data from the member nodes. In order to minimize the data to be transmitted and improve the compression ratio, a threshold (T), such that $\mathrm{v}=T / \hat{M}$, is applied at the one hop neighbours of the $\mathrm{FCH}$.

Sensor nodes in each cluster, on detecting an event, transmit their readings to their respective FCHs through one hop or two hop transmission only. If the incoming traffic, at the one hop neighbours, increases from the predefined threshold (T), the received data is compressed using the CS principle. Application of CS at this point of the network not only minimizes the network traffic but also imposes no extra load on the intermediate nodes.

Otherwise, the received data is transmitted to FCHs without compression. CS at one hop neighbours is applied in the same way as on the FCH's and is explained later in this section. One of the FCHs is designated as the $\mathrm{SCH}$ and receives the data forwarded by all the FCHs. It is assumed that each $\mathrm{FCH}$ already knows the value of the projection vectors in the measurement matrix $\Phi$ for all the nodes that belong to that cluster. In real environment a pseudorandom number generator is used to generate the value of the measurement coefficient $\Phi$ using the unique id of every node. Thus, with the node id's known, the measurement matrix is constructed locally at the sink and at the cluster heads. Sub-matrices for respective clusters are formed at each $\mathrm{FCH}$, by decomposing the measurement matrix $\Phi$. Let the sub-matrix for $i^{\text {th }}$ cluster is given by $\Phi^{\mathrm{CHi}}$, the respective cluster head is given by $\mathrm{CH}_{i}$, and the cluster's data vector is given by $d^{C H i}$. The cluster head, $\mathrm{CH}_{i}$, computes the projection of all the data items within the cluster by multiplying the measurement matrix for the cluster with the data received from all its nodes, that is $\Phi^{C H i} d^{C H i}$.

Finally, M projections of the cluster data is forwarded by the $\mathrm{CH}_{i}$ to the $\mathrm{SCH}$. The number of nodes in the cluster and the sparsity of the data determines the value of $\mathrm{M}$. Each $\mathrm{SCH}$, adds its own data (i.e. data of its own cluster) with the data obtained from all the members of the first level cluster and apply Huffman encoding on the received data.

The encoded data is then forwarded to the sink. The sink is responsible for recovering the original data from the received samples. At the sink, Huffman decoding algorithm is used to recover the compressed data sent to the $\mathrm{SCH}$ followed by $\mathrm{L} 1$ magic for recovering the original data. 

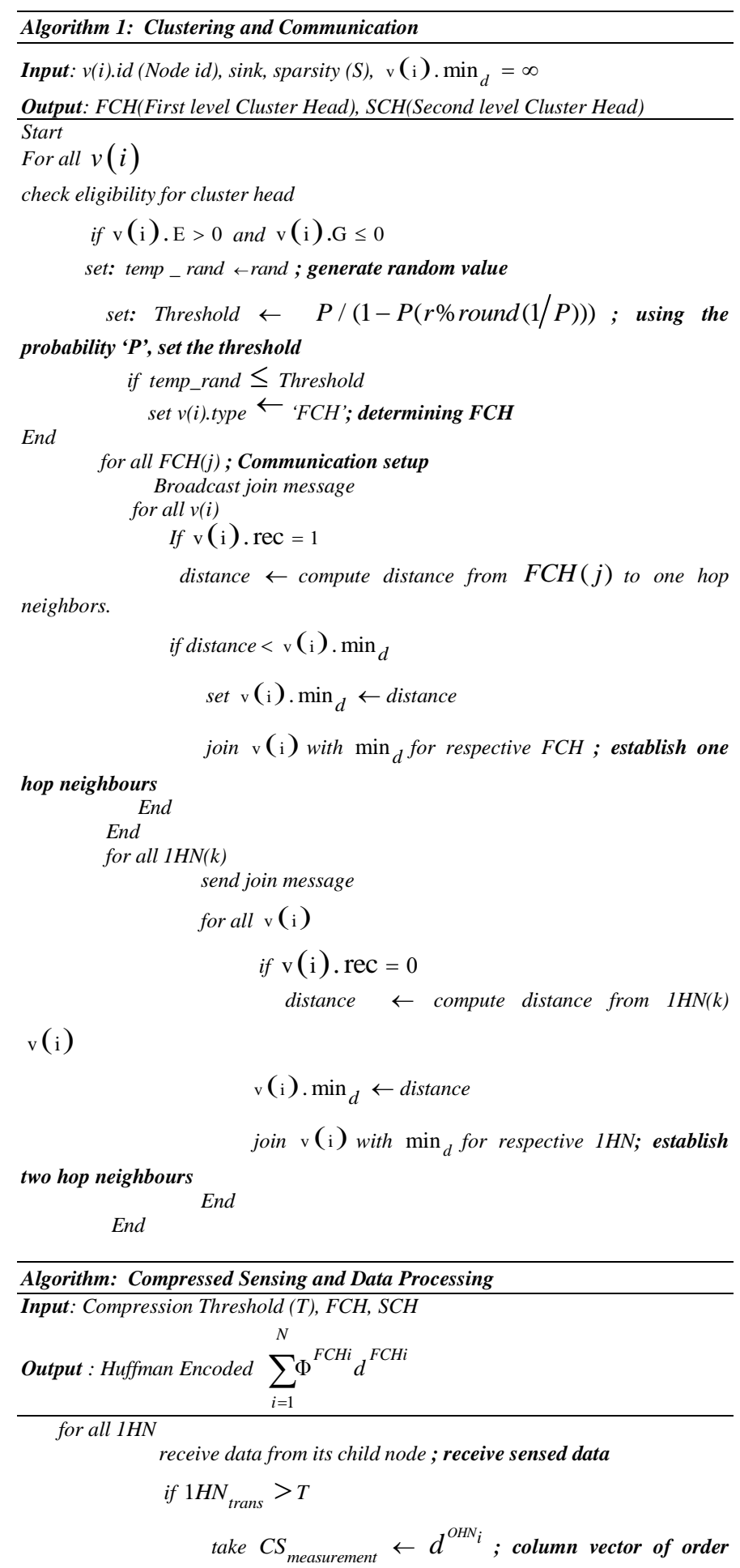

$(N \times 1)$

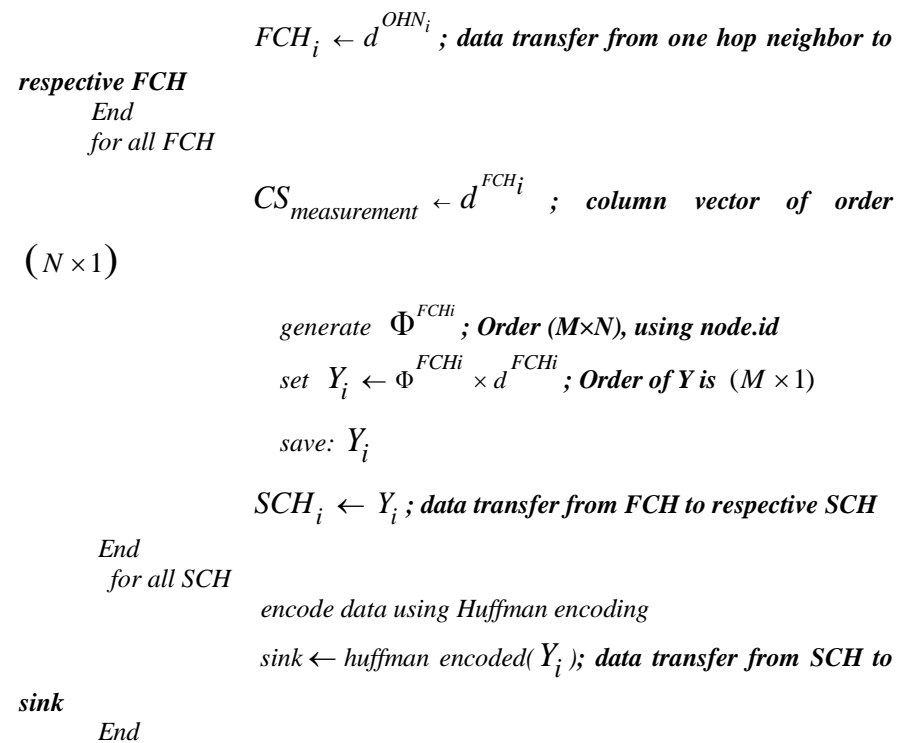

TABLE I. SiMULATION PARAMETERS

\begin{tabular}{|c|c|}
\hline Parameter & Value \\
\hline Network size & $100 m \times 100 m$ to $500 m \times 500 m$ \\
\hline Number of sensor node (n) & 100 (minimum) and 900 (maximum) \\
\hline Sink position & $(200 \mathrm{~m}, 250 \mathrm{~m})$ and $(100 \mathrm{~m}, 100 \mathrm{~m})$ \\
\hline Initial energy & $0.5 \mathrm{j}$ \\
\hline $\begin{array}{l}\text { Transmitter/Receiver } \\
\text { electronics }\left(E_{\text {elec }}\right)\end{array}$ & $50 \mathrm{nj} / \mathrm{bit}$ \\
\hline Data aggregation ( $E_{D A}$ ) & $5 \mathrm{nj} / \mathrm{bit} / \mathrm{report}$ \\
\hline Transmit amplifier $\left(\grave{O}_{\mathrm{fs}}\right)$ & $10 \mathrm{pj} / \mathrm{bit} / \mathrm{m} 2$ \\
\hline $\begin{array}{l}\text { Sparse ratio of projection } \\
\text { matrix }(\Phi)\end{array}$ & $\log _{2}(N) / N$ \\
\hline Message size $(l)$ & 1024 bits \\
\hline
\end{tabular}

\section{RESULTS AND DISCUSSION}

To evaluate the performance of the proposed framework, simulations were performed for different scenarios and the outcomes were compared with different existing CS based data gathering schemes [23], [24] and [25].

\section{A. Simulation Setup}

Lifetime of the network and the number of transmissions, in the proposed scheme, are tested with varying sink positions and are compared with the results presented in [23], [24] and [25]. Table I describes the parameters used for simulations.

\section{B. Communication and Load Distribution analysis}

The proposed HCS aims at optimizing the energy consumption by even distribution of the load over the network and maximizing the lifetime of the network by reducing the number of transmissions. The energy consumption at every node is mainly because of the following two kind of activities:

- Transmissions received - A major portion of energy is utilized in receiving the bits from other connected 
nodes in the network. Hence, the total number of transmissions received at every node is monitored and the activity of every node is mapped for each round of data transmission.

- Transmissions sent - The received packets are processed and are forwarded to the next hop. The energy dissipated in processing the data is minimal and hence the data forwarding is the next major energy consuming task.

Interestingly, in-network compression using CS allows minimum energy consumption for compression. The major energy consuming task being the recovery of the compressed data, is done at the sink which has sufficient energy resources. The proposed HCS exploits the advantages of CS in such a way that the load is evenly distributed over the network and the number of transmissions between the sensor nodes is minimum. Node activities are monitored and mapped for the following scenarios:

\section{Sink located outside}

The Fig. 3 shows a random deployment of 400 sensor nodes in $200 m \times 200 m$ area. The sink is located at a corner outside the deployment area. The activity of each node is monitored for each data gathering round and is mapped for the number of transmissions in every round. The activity of each node in one such round, specifically for the deployment shown in Fig. 3, is shown in the activity map 1.

As seen in the Fig. 3, the sink is located at the right corner $(200,250)$ while the nodes are randomly deployed in an area of $200 m \times 200 m$. While the $\mathrm{FCH}(\mathrm{FCH})$ are many the $\mathrm{SCH}$ is only one for this round and is located at $(81.78,188.90)$. The number of transmissions i.e. sent and received, for every node is analysed and mapped to activity map shown in activity map 1(a) and activity map 1(b) respectively.

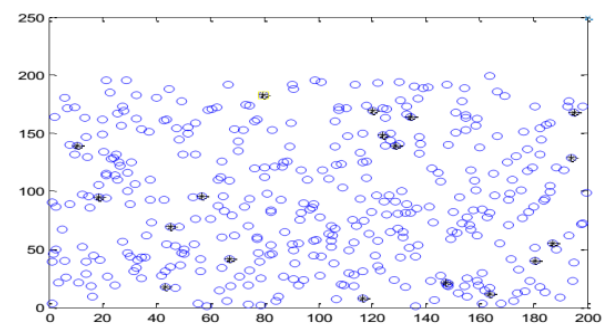

Fig. 3. Random deployment of nodes in $200 m \times 200 m$ area (sink outside)

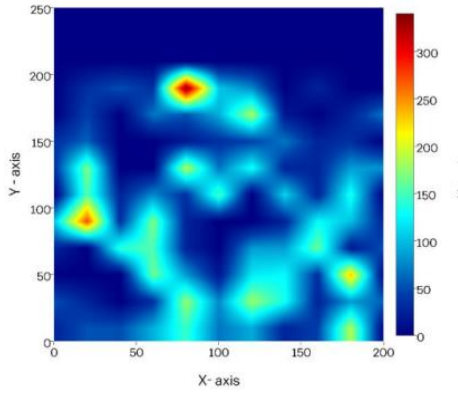

(a)

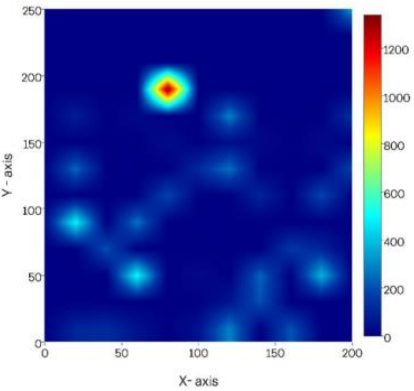

(b)
Activity map 1: Activity of nodes (a) sending transmissions (b) receiving transmissions
As seen in the activity map 1(a), data sending activity is marked all around the map leading to the mapping of moderate transmissions (between 80 and 135) by the sensor nodes at different locations in the map. The two dark patches around $(81.78,188.90)$ and $(22.24,90.01)$ signify relatively higher activity. The reason for this high activity around (81.78, 188.90) can be understood from the fact that the proposed HCS allows only the SCHs to communicate with the sink. Since, for this deployment, the $\mathrm{SCH}$ is located at $(81.78,188.90)$ hence it is responsible for sending all the information obtained from its member nodes. This distribution of ability to communicate with the sink allows all the other nodes to save a lot of energy which would have been dissipated by them otherwise. Huge activity is seen around $(22.24,90.01)$ because the FCH located at this position is the only one in the region and is responsible for sending the data from a large number of nodes. Moderate patches over the map show low number of transmissions (between 80 and 135) between the FCHs and respective SCHs. The sink at the corner shows no activity as it only receives data from the $\mathrm{SCH}$. An important conclusion that can be drawn from this map is that the number of transmissions at every node is moderate and varies between 100 and 150. As seen in the activity maps every node shares the load equally and hence the number of sending activity along with the transmission load on every node is distributed evenly throughout the network.

As seen in the activity map 1(b), data receiving activity is marked all around the map leading to the mapping of moderate transmissions (between 400 and 500) by the sensor nodes at different locations in the map. It must be noticed that moderate receiving activity is seen at all the one hop neighbours and FCHs. The proposed HCS ensures that the number of received transmissions at every node is reduced to a low value (around 400 in this case) except at the SCH. The reduced amount of receiving activity at various $\mathrm{FCH}$ shows the advantage of applying $\mathrm{CS}$ at the one hop neighbours. The SCH, at $(81.78$, 188.90), shows the highest number of receiving activity for obvious reasons as it receives data from all the FCHs. As seen in the activity map $1(\mathrm{~b})$, the $\mathrm{SCH}$ receives about 1200 transmissions but careful observation reveals that the sink at the corner $(200,250)$ receives about 300 transmissions only. Comparing the activity maps 1(a) and (b), it becomes clear that although the second level cluster receives a large number of transmissions (in this case about 1200) but with the proposed HCS, the outgoing traffic is reduced to nearly 300 . With such a distribution of transmissions, the nodes save a lot of energy and hence the network lifetime is enhanced significantly. A significant deduction from this activity map is that the application of CS at one hop neighbours and at FCHs prevents the nodes from transmitting huge amount of data within the network. Thus, the activity maps proves that with reduced number of transmissions the proposed HCS can enhance the network lifetime significantly.

\section{Sink located at the centre}

The Fig. 4 shows a random deployment of 400 sensor nodes in $200 m \times 200 m$ area. The sink is located at the centre of the deployment area. The activity of each node is monitored for each data gathering round and is mapped for the number of transmissions in every round. The activity of each node in one 
such round, specifically for the deployment shown in Fig. 4, is shown in the activity map 2 .

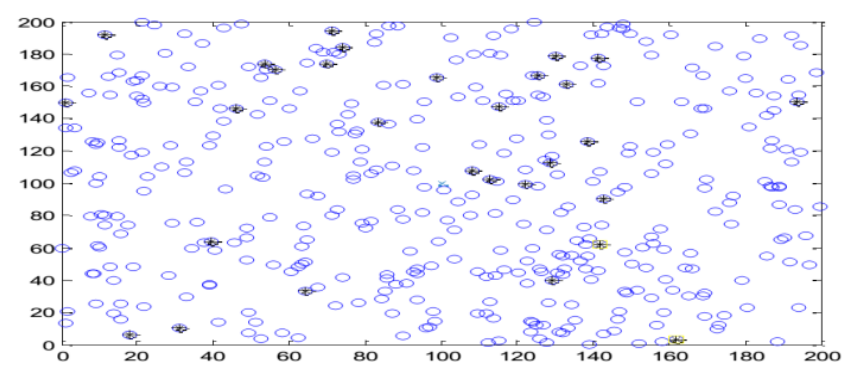

Fig. 4. Random deployment of nodes in $200 m \times 200 m$ area (sink outside)

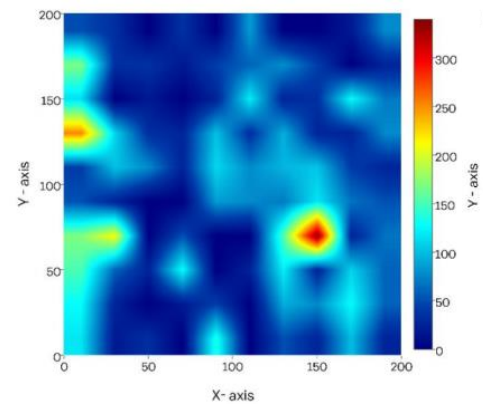

(a)

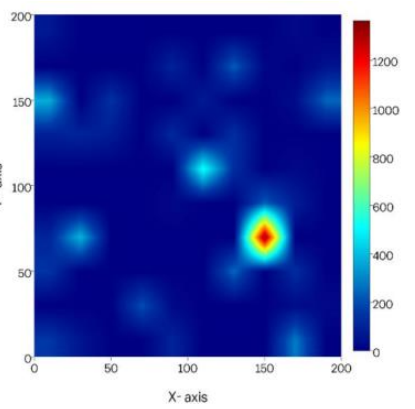

(b)
Activity map 2: Activity of nodes (a) sending transmissions (b) receiving transmissions

As seen in the activity map 2(a), data sending activity is marked all around the map leading to the mapping of moderate transmissions (between 55 and 110) by the sensor nodes at different locations in the map. The two dark patches around $(144.56,62.76)$ and $(0,151.01)$ signify relatively higher activity. The reason for this high activity around (144.56, 62.76) is the presence of SCH. Since the SCH sends majority of the data to the sink, the number of transmissions being sent by the $\mathrm{SCH}$ is relatively high (about 300 in this case). A small patch of relatively lower transmissions is seen around (161.08, 2.23) which is the location of the second SCH. The activity at this location is moderate because as seen in the deployment diagram (figure 6) the majority of the $\mathrm{FCHs}$ lie closer to (144.56, 62.76) and hence they send their data to it. Thereby leaving only a few $\mathrm{FCH}$ to send their data through the $\mathrm{SCH}$ at $(161.08,2.23)$, thus less data to send to the sink. Huge activity is seen around $(0,151.01)$ because the FCH located at this position is the only one in the region and is responsible for sending the data from a large number of nodes. A patch with moderate activity is seen around $(50,62.7)$ as there are relatively less number of $\mathrm{FCHs}$ in the region and hence increased two hop transmissions. The sink at the centre shows no activity as it only receives data from the SCHs. As compared to the activity map 1(a) the nodes in the activity map 2(a) have lower transmission values ranging between 55 and 110 and the load is more evenly distributed throughout the area. The advantage of having the sink at the centre of the deployment area is the significant reduction in the transmission distance for the $\mathrm{SCH}$. With almost equal activity at every node the load is evenly distributed throughout the area. With sink at the centre the results tend to improve and the effect of this reduction is seen in the lifetime of the network. Another round of data gathering might result in different number of FCHs and SCHs with different positions.

As seen in the activity map 2(b), data receiving activity is marked all around the map leading to the mapping of moderate transmissions (between 350 and 500) by the sensor nodes at different locations in the map. Important deduction from this activity map is the better distribution of the transmission load as compared to the activity map 1(b) for sink outside the deployment area. The SCH, at $(144.56,62.76)$, shows the highest number of receiving activity as it receives data from all the majority of the FCHs. As discussed in activity map 2(a) the $\mathrm{SCH}$ at $(161.08,2.23)$ shows relatively low receiving activity as majority FCHs are near to the SCH at (144.56, 62.76). The receiving activity at the sink shows that the number of transmissions received at the sink is almost same as in activity map 1(b) but the difference being the distance between the SCHs and the sink. With sink at the centre, the energy consumption in transmitting the data is relatively low and hence is much better.

\section{Transmission Analysis}

The sink position remaining the same i.e. at the centre, the total number of packets circulating within the network for the proposed HCS is compared with the mixed algorithm proposed in [23]. The performance of the proposed algorithm is also compared with the other approaches i.e. Distributed compressed sensing (DCS) and Pack and forward (PF) strategies as used in [23]. The Fig. 5 shows the behaviour of the proposed HCS and the three existing approaches as the nodes are increased from 9 to 900 .

The figure shows a gradual increase in the number of transmissions as the number of nodes is increased. However, Fig. 5 (a) clearly shows that with the proposed HCS, the number of packets within the network is far less as compared to the mixed algorithm proposed in [23] and DCS and PF schemes used for comparison in [23]. Even for a small network, the number of packets in the proposed HCS is much better than the mixed algorithm. Interestingly, a sudden burst, in the number of sent packets, is seen for a particular network size in the mixed algorithm, DCS and PF scheme. However, the proposed HCS remains unaffected with the network size, proving its efficiency over the existing approaches. As seen in Fig. 5 (b), the average number of transmitted packets per node ranges between 0.1175968 and 0.58376 which is much better than the mixed algorithm [23] where the range of per node packet ranges approximately between 0.333856 to 1.1625 . The proposed HCS has better performance than the existing approaches, presenting very less number of sent packets which is always better than the mixed algorithm [23], DCS and PF as used in [23].

\section{Energy and Network Lifetime analysis}

The lifetime of the network is analyzed for both the discussed scenarios. The stability period, first dead and final dead are considered as the parameters for the energy and lifetime analysis.

\section{Sink located outside}

The Fig. 6 shows the lifetime of the network with the proposed HCS, with sink outside the deployment area. 


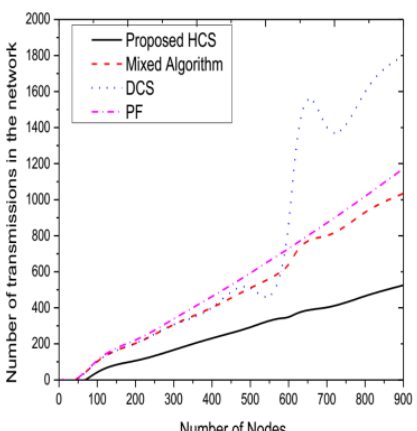

(a)

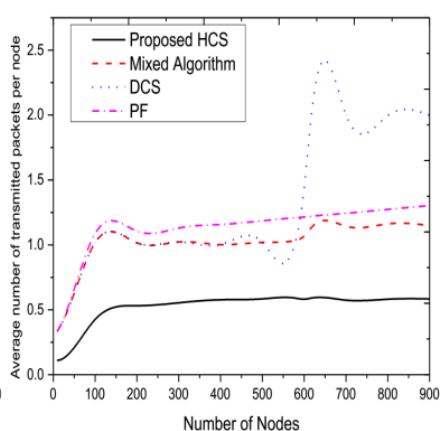

(b)
Fig. 5. Comparison among proposed HCS, mixed algorithm, DCS and PF (sink at the centre). (a) Total number of packets circulating within the network. (b) Average number of transmissions per node

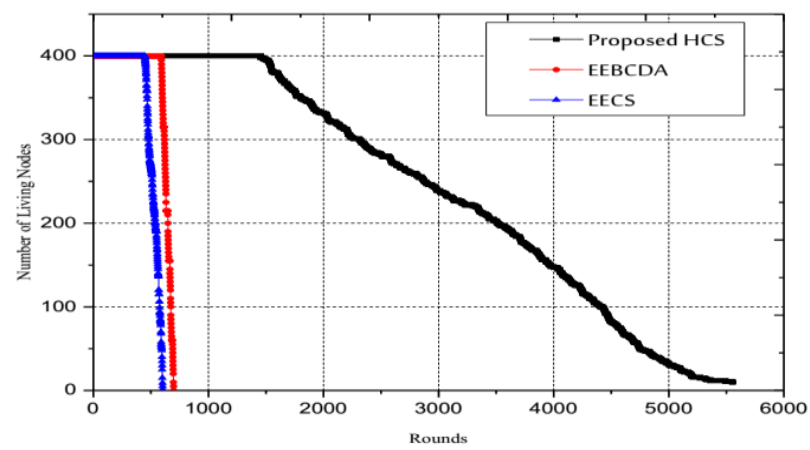

Fig. 6. The number of living nodes over rounds (sink outside)

As evident from the Fig. 6, the proposed HCS is able to improve the lifetime of the network to almost $200 \%$ as compared to EEBCDA as proposed in [24]. The first node with the proposed HCS dies in the $1362^{\text {nd }}$ round whereas with EEBCDA [24] the first node dies in the $591^{\text {st }}$ round. The proposed HCS outperforms the EECS [25] and EEBCDA [24], both in terms of stability period and lifetime of the network. The simulations are run until only 10 nodes are alive and, with the proposed scheme, the $390^{\text {th }}$ node dies in the $5380^{\text {th }}$ round after which the network is considered to be dead. An important aspect of EEBCDA [24] and EECS [25] is the even distribution of the load over the network. The relatively small unstable region i.e. the duration between the first and the last dead, in EEBCDA [24] signifies efficient traffic load balancing within the network. However, in the proposed HCS, with sink at the corner outside the deployment area, the SCHs spend huge amount of energy in transmitting their data to the sink. Over the lifetime of the network, every node becomes a $\mathrm{SCH}$ and bears this heavy energy consumption. Hence, although the load is distributed efficiently for almost all the nodes in the network, there might be one or more (depending upon the number of $\mathrm{SCHs}$ ) nodes dissipating huge amount of energy in data transmission to the sink. The presence of such nodes, in every round of data collection, does not allow the proposed HCS to achieve its true potential in terms of stability period and lifetime. The effect is seen in the duration between the first and the last dead of the proposed HCS. After the first node dies in the $1362^{\text {nd }}$ round, the last considered alive node i.e. $390^{\text {th }}$ node dies in the $5380^{\text {th }}$. Thus, in the current scenario, though the lifetime of the network is improved greatly but the advantage of distributing the load throughout the network is lost.
Changing the position of the sink can not only facilitate the load distribution in the network but can also improve the stability period of the network.

\section{Sink located at the centre}

The Fig. 7 shows the lifetime of the proposed HCS with sink at the centre.

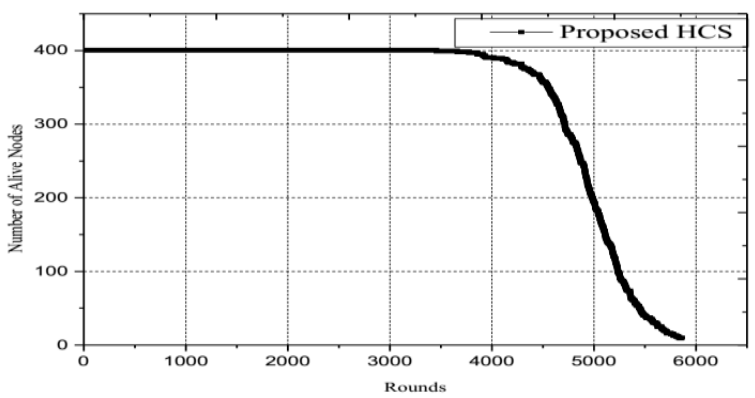

Fig. 7. The number of living nodes over rounds (sink at centre)

As evident from the figure, with sink at the centre, not only a perfect distribution of the load is obtained but the stability period is doubled as well. In the current scenario, the first node dies in the $3873^{\text {rd }}$ round whereas when the sink is placed outside the deployment area, the first node dies in the $1362^{\text {nd }}$ round. The proposed HCS, in the current scenario, is able to improve the stability period of the network to almost $200 \%$ as compared to the scheme proposed in scenario 1 . The simulations are run until 10 nodes are alive and the $390^{\text {th }}$ node dies in the $5878^{\text {th }}$ round and after this the network is considered to be dead. Comparing the lifetime of EEBCDA proposed in [24] with the proposed HCS (both scenarios), it becomes clear that the proposed HCS (with sink at the centre) improves the lifetime of the network significantly and is able to achieve about $300 \%$ efficiency over EEBCDA [24] and is even better than EECS [25]. With sink at the centre, the proposed HCS is able to efficiently distribute the load throughout the network and hence better traffic load balancing in the network. The effect of this even distribution is seen in the reduced unstable region. Thus, in the current scenario, not only the advantage of distributing the load throughout the network is achieved but the stability period is also improved significantly. Changing the position of the sink to the centre allows the proposed HCS to achieve better results both in terms of network lifetime and reliability as well.

\section{CONCLUSION}

This paper addressed the problem of network lifetime and uneven energy consumption in large scale WSNs. A Hierarchical Compressed Sensing (HCS) scheme is proposed to achieve efficient traffic load balancing and reduced innetwork communication. A major concern for the existing CS based methods is the point of application of CS and this has been successfully resolved in the proposed scheme. HCS is able to reduce and uniformly distribute the number of transmissions in the network. The effect is seen in the lifetime of the network which grows significantly as compared to the existing approaches. Due to efficient load distribution among the nodes of the WSN the availability of all the nodes for data gathering is increased significantly i.e. the stability period is 
improved. The same has been validated through results. The results prove that the proposed HCS outperforms the existing CS based schemes.

\section{FUTURE WORK}

We continue to extend our work to analyse the performance of the proposed HCS in a real world deployment. A mathematical model to determine the compression ratio and the communication overhead, remains to be developed in the future.

\section{REFERENCES}

[1] Abbasi AA, Younis M. A survey on clustering algorithms for wireless sensor networks. Computer communications. 2007 Oct 15;30(14):282641.

[2] Singh S, Chand S, Kumar B. Energy Efficient Clustering Protocol Using Fuzzy Logic for Heterogeneous WSNs. Wireless Personal Communications. 2016 Jan 1;86(2):451-75.

[3] Xue Y, Cui Y, Nahrstedt K. Maximizing lifetime for data aggregation in wireless sensor networks. Mobile Networks and Applications. 2005 Dec 1;10(6):853-64.

[4] Tan HÖ, Körpeoğlu I. Power efficient data gathering and aggregation in wireless sensor networks. ACM Sigmod Record. 2003 Dec 1;32(4):6671.

[5] Sinha A, Lobiyal DK. A multi-level strategy for energy efficient data aggregation in wireless sensor networks. Wireless personal communications. 2013 Sep 1;72(2):1513-31.

[6] Banerjee T, Chowdhury KR, Agrawal DP. Using polynomial regression for data representation in wireless sensor networks. International Journal of Communication Systems. 2007 Jul 1;20(7):829-56.

[7] Othman SB, Bahattab AA, Trad A, Youssef H. Confidentiality and Integrity for Data Aggregation in WSN Using Homomorphic Encryption. Wireless Personal Communications. 2015 Jan 1;80(2):86789.

[8] Liu T, Li Q, Liang P. An energy-balancing clustering approach for gradient-based routing in wireless sensor networks. Computer Communications. 2012 Oct 1;35(17):2150-61.

[9] Sutagundar AV, Manvi SS. Wheel based Event Triggered data aggregation and routing in Wireless Sensor Networks: Agent based approach. Wireless Personal Communications. 2013 Jul 1;71(1):491517.

[10] Khedo K, Doomun R, Aucharuz S. Reada: Redundancy elimination for accurate data aggregation in wireless sensor networks. Wireless Sensor Network. 2010 Apr 1;2(4):300.

[11] Guo W, Xiong N, Vasilakos AV, Chen G, Cheng H. Multi-source temporal data aggregation in wireless sensor networks. Wireless personal communications. 2011 Feb 1;56(3):359-70.

[12] Enachescu M, Goel A, Govindan R, Motwani R. Scale free aggregation in sensor networks. InAlgorithmic Aspects of Wireless Sensor Networks 2004 Jul 16 (pp. 71-84). Springer Berlin Heidelberg.

[13] Luo J, Xiang L, Rosenberg C. Does compressed sensing improve the throughput of wireless sensor networks?. InCommunications (ICC), 2010 IEEE International Conference on 2010 May 23 (pp. 1-6). IEEE.
[14] Razzaque MA, Dobson S. Energy-efficient sensing in wireless sensor networks using compressed sensing. Sensors. 2014 Feb 12;14(2):282259.

[15] Xiang L, Luo J, Vasilakos A. Compressed data aggregation for energy efficient wireless sensor networks. InSensor, mesh and ad hoc communications and networks (SECON), 2011 8th annual IEEE communications society conference on 2011 Jun 27 (pp. 46-54). IEEE.

[16] Caione C, Brunelli D, Benini L. Compressive sensing optimization over ZigBee networks. InSIES 2010 Jul 7 (pp. 36-44).

[17] Mehrjoo S, Shanbehzadeh J, Pedram MM. A novel intelligent energyefficient delay-aware routing in wsn, based on compressive sensing. InTelecommunications (IST), 2010 5th International Symposium on 2010 Dec 4 (pp. 415-420). IEEE.

[18] Zheng H, Xiao S, Wang X, Tian X. On the capacity and delay of data gathering with compressive sensing in wireless sensor networks. InGlobal Telecommunications Conference (GLOBECOM 2011), 2011 IEEE 2011 Dec 5 (pp. 1-5). IEEE.

[19] Zheng H, Xiao S, Wang X, Tian X, Guizani M. Capacity and delay analysis for data gathering with compressive sensing in wireless sensor networks. Wireless Communications, IEEE Transactions on. 2013 Feb;12(2):917-27.

[20] Xu X, Ansari R, Khokhar A. Power-efficient hierarchical data aggregation using compressive sensing in WSNs. InCommunications (ICC), 2013 IEEE International Conference on 2013 Jun 9 (pp. 17691773). IEEE.

[21] Chung WY, Villaverde JF. Implementation of Compressive Sensing Algorithm for Wireless Sensor Network Energy Conservation. InInternational Electronic Conference on Sensors and Applications 2014. Multidisciplinary Digital Publishing Institute.

[22] Qaisar S, Bilal RM, Iqbal W, Naureen M, Lee S. Compressive sensing: From theory to applications, a survey. Communications and Networks, Journal of. 2013 Oct;15(5):443-56

[23] Caione C, Brunelli D, Benini L. Distributed compressive sampling for lifetime optimization in dense wireless sensor networks. Industrial Informatics, IEEE Transactions on. 2012 Feb;8(1):30-40.

[24] Yuea J, Zhang W, Xiao W, Tang D, Tang J. Energy efficient and balanced cluster-based data aggregation algorithm for wireless sensor networks. Procedia Engineering. 2012 Dec 31;29:2009-15.

[25] Ye M, Li C, Chen G, Wu J. EECS: an energy efficient clustering scheme in wireless sensor networks. InPerformance, Computing, and Communications Conference, 2005. IPCCC 2005. 24th IEEE International 2005 Apr 7 (pp. 535-540). IEEE.

[26] Chand S, Singh S, Kumar B. Heterogeneous HEED protocol for wireless sensor networks. Wireless personal communications. 2014 Aug 1;77(3):2117-39.

[27] Davenport MA, Duarte MF, Eldar YC, Kutyniok G. Introduction to compressed sensing. Preprint. 2011;93(1):2.

[28] Han Z, Li H, Yin W. Compressive sensing for wireless networks. Cambridge University Press; 2013 Jun 6.

[29] Razzaque MA, Bleakley C, Dobson S. Compression in wireless sensor networks: A survey and comparative evaluation. ACM Transactions on Sensor Networks (TOSN). 2013 Nov 1;10(1):5.

[30] Donoho DL. Compressed sensing. Information Theory, IEEE Transactions on. $2006 \mathrm{Apr} ; 52(4): 1289-306$. 